\title{
The case for a cohort
}

\author{
Public health needs to be turned over to the public. Participation in genome-enabled research into the determinants \\ of health is an important form of participation in society and a secure way to ensure the constant improvement of \\ healthcare delivery.
}

$\mathrm{H}$ ealthcare is not just consumed. It is subsidized and improved by the public in various ways and at varying levels of enthusiasm, from universal participation to the extraordinary efforts of rare volunteers. As a responsible citizen, you seek vaccination of yourself and your children to prevent epidemic disease in other members of your society. As a registered blood donor, the regular, healthy operation of your body contributes to the healthcare infrastructure where you live. As a stem cell donor, you can respond to an infrequent call for matching bone marrow. As a registered organ donor, even your death in a catastrophic accident can bring a life-saving transplant to someone else in need. You are part of a living cohort without whose regular efforts the delivery of healthcare would grind to a halt.

However, even with your efforts and increasing payments by yourself, your employer and your government, healthcare improvement is indeed slowing. There are few new drugs in the pipeline, and these are increasingly expensive to license. People are living longer with chronic and degenerative conditions as well as with expectations of rising costs of care. Gaps in access to healthcare between rich and poor people, both within and between countries, are widening. At the same time, public groups concerned with single diseases are marching and raising money and awareness but are becoming frustrated with the perceived slow pace of research and translation.

One solution is public participation by 'health donors', people who are prepared to provide full information about their genotypes in health and disease. Enabling this form of participation requires incentives. Current protections are not well suited to incentivize volunteers, as research is not a simple matter of taking participants' data and locking it up securely for the use of experts. Rather, incentives should encourage participants to remain engaged in improving healthcare. All forms of 'omic' information are inherently excellent ways to identify individuals, and we must be honest in communicating this and planning for its consequences. Indeed, most forms of linked data can be used to unearth personal and private information (Nature 497, 172-174, 2013). As with other identifying information, the law ought not to penalize those responsible for data collection and storage but rather those responsible for discrimination, misrepresentation and other negative uses of personal data, including genome sequences, that have been appropriately volunteered for the public good. In most cases, these abuses are theoretical worst-case outcomes, but fears of loss of employment, insurance and reputation are serious disincentives to participation.

In our editorial last June, 'Asking for more' (Nat. Genet. 44, 733, 2012), we emphasized that the progress made in genetic epidemiol- ogy will only continue if the publication strategy includes a commitment to make research papers more useful to other researchers in three respects: greater access to individual-level genotype-phenotype information, discussion of the full spectrum of rare and common variation and an attempt to interpret trait-associated variants in the genomic context of elements regulating gene expression. Now, on page 580, the GTEx Consortium describes its strategy to interpret the consequences of regulatory SNPs in a variety of human tissues, creating a large catalog of expression quantitative trait loci (eQTLs). The research participants in this study are volunteers who are properly consented, but-because of the requirements for human tissue-most are deceased at the time of tissue collection. The study is likely to make important contributions to the translation of genome-wide association study (GWAS) results for medicine, so it is not too early to find appropriate populations in whom to test genomic predictors of disease susceptibilities and to design genomically informed clinical trials.

Genomics is global, so there is no reason health donor cohorts could not be established anywhere, but we think a good place to start is in Europe, as the first results from GWAS came from genetic epidemiology of European populations. Now is a good time to discuss priorities because there is a large tranche of the proposed European Union (EU) Horizon 2020 framework funding package devoted to health, demographic change and well-being (http://ec.europa.eu/research/ horizon2020/index_en.cfm). Within this element are aims of understanding what determines health, how to screen and survey populations and assess disease susceptibility, and how to prepare for environmental and epidemic challenges, understand disease and promote prevention, healthcare innovation and better use of health-related data, including genomics.

It is also time to consider which populations should take the lead. Investment in health research should already be integral to the planning of public-health policy. Statistical considerations require sufficient population size, so like-minded nations might pool resources. Epidemiological considerations favor local populations and long-ranging, detailed record keeping. Open data sharing within the bounds of local laws and customs is likely to be most productive, but there is no need for the project to be open to the world. Local sharing should return benefit to the participating populations first, and their example can then be copied. Measures of success for refunding and scaling up the experiment will be effective data sharing, the translation of research results into improvements in clinical delivery and the scheduling of clinical trials in the participating populations. 